\title{
Estructuras metal-orgánicas (MOFs) nanoestructuradas para la liberación controlada de fármacos
}

\section{Nanostructured metal-organic frameworks (MOFs) applied to controlled drug delivery}

\author{
Jesús A. Claudio-Rizo, ${ }^{*}$ Lucia F. Cano Salazar, ${ }^{*}$ Tirso E. Flores-Guia, ${ }^{*}$ \\ Denis A. Cabrera-Munguia***
}

\begin{abstract}
Metal-organics frameworks are coordination polymers constituted of a metallic center and an organic ligand that can be designed using biocompatible metal ions (e.g. $\mathrm{Fe}^{+3}, \mathrm{Zn}^{+2}$, $\mathrm{Mg}^{+2}, \mathrm{Ca}^{+2} \mathrm{y} \mathrm{Mo}^{+6}$ ) and bioactive organic ligands yielding to the modulation of the structure and properties materials in the nano scale. The aim of this work is provide an outlook about the materials applied for drug delivery systems, emphasizing the advantages, advances and challenges in the controlled drug delivery using nanostructured MOFs, the delivery mechanism, degradability and antimicrobial activity is also presented.
\end{abstract}

KEYWORDS: MOFs, nanostructure, controlled drug delivery, biocompatibility.

RESUMEN: Las estructuras metal-orgánicas (MOFs) son polímeros de coordinación que se componen por un centro metálico y un ligando orgánico, que pueden diseñarse empleando iones metálicos biocompatibles $\left(\mathrm{Fe}^{+3}, \mathrm{Zn}^{+2}, \mathrm{Mg}^{+2}, \mathrm{Ca}^{+2}\right.$ y $\mathrm{Mo}^{+6}$ ) y ligandos orgánicos bioactivos, permitiendo la modulación de la estructura y propiedades a escala nanométrica. El objetivo del presente trabajo es dar a conocer un panorama de los materiales empleados en la liberación de fármacos, enfatizando las ventajas, avances y retos en la liberación de fármacos con MOFs nanoestructurados, así como su mecanismo de liberación, degradación y actividad antimicrobiana.

PALABRAS CLAVE: MOFs, nanoestructura, liberación controlada de fármacos, biocompatibilidad.

\section{Introducción}

La nanotecnología y la nanomedicina se han enfocado en el desarrollo de materiales capaces de almacenar y liberar de manera controlada moléculas terapéuticas. En este sentido, la biomedicina se ha inspirado en estudiar el efecto de la estructura y la naturaleza que este tipo de materiales exhiben en la modulación de la respuesta celular, asociada con procesos fisiológicos y/o patológicos involucrados en una enfermedad. El principal objetivo de la liberación controlada de fármacos es la transferencia del mismo a un tejido, órgano o grupo de células específico, sin dañar o causar efectos secundarios en regiones sanas del organismo. Para lograrlo, es necesario conocer los efectos de la solubilidad y toxicidad del fármaco, así como los productos de degrada-

* Universidad Autónoma de Coahuila, Facultad de Ciencias Químicas, Saltillo, Coahuila, México.

- Autora de correspondencia: dcabrera@uadec.edu.mx 
ción del vehículo liberador sobre la respuesta celular, evitando así que los procesos de crecimiento, división e interacción celular; rutas de señalización hormonal y regulación metabólica sean afectados negativamente (Rasheed et al., 2019).

Hasta el momento se han empleado nanopartículas inorgánicas metales (Paul y Sharma, 2010), óxido de hierro (Anirudhan y Christa, 2020), sílice mesoporosa (Angelos et al., 2008) y nanotubos de carbono (Kaur et al., 2019), así como liposomas (Bruch et al., 2019), dendrímeros (Sherje et al., 2018) y nanopartículas orgánicas a base de polímeros sintéticos y biopolímeros (celulosa, quitosano, ciclodextrina, colágeno, gelatina) (Barclay et al., 2019). La escala nano de estas partículas facilita su difusión dentro de las células, permitiendo la internalización del material y la posterior liberación del fármaco contenido. El principal problema de las partículas inorgánicas es la liberación total del fármaco dentro de las células objetivo (ej. células de un tumor), debido a su baja permeabilidad, nula estabilidad coloidal y bajo tiempo de retención en el sistema circulatorio.

Lo ideal sería poder combinar las propiedades de las nanopartículas inorgánicas como porosidad, área superficial, estabilidad mecánica, propiedades ópticas y magnéticas, junto con las propiedades de baja toxicidad, biodegradabilidad, estabilidad coloidal y control de la solubilidad en agua de nanopartículas orgánicas (Couvreur, 2012; Li Chong et al., 2019; Wang et al., 2019).

Una potencial alternativa a esta problemática, lo presentan las estructuras metal orgánicas (MOFs), una clase de materiales con una estructura cristalina ordenada, tamaño de poro modulable y una gran área superficial; lo que le otorga un amplio potencial de aplicación en los campos de adsorción y separación, catálisis, sensores, imagenología biológica y liberación de fármacos (Horcajada et al., 2010). Los MOFs nanoestructurados presentan características ideales como área superficial y propiedades dependientes del tamaño de partícula, que podrían ser aprovechadas para la adsorción de moléculas con interés terapéutico y ser liberadas al internalizarse dentro de células objetivo. Con esto en mente, algunas de las ventajas que ofrecen los MOFs son una alta capacidad de almacenamiento del fármaco asociada con su nanoestructura, biocompatibilidad dependiente de la composición y la capacidad de regular la liberación de los terapéuticos encapsulados modificando la fuerza iónica, temperatura y $\mathrm{pH}$ del medio exhibiendo perfiles controlados y sostenibles, potencializando la eficacia de los fármacos. A pesar de haberse sintetizado un sinnúmero de MOFs, solo algunos han podido ser aplicados en la liberación de fármacos, al demostrar tener una alta capacidad de almacenamiento, un efecto prolongado de liberación de fármacos y estabilidad en un medio acuoso fisiológico (Liu Chong et al., 2019). El presente artículo de revisión detalla las principales características de los MOFs necesarias para la liberación controlada de fármacos, incluyendo su síntesis, funcionalización y recubrimiento, el mecanismo de liberación de fármacos, de- 
gradación y toxicidad; así como los avances e investigación futura en esta relativamente nueva área del conocimiento.

\section{Liberación controlada de fármacos}

Básicamente existen dos sistemas de liberación de fármacos: los convencionales y los sistemas de liberación controlada. Los fármacos convencionales pueden ser liberados de manera oral, bucal/sublingual, rectal, intravenosa, subcutánea e intramuscular. En estos métodos la concentración de los agentes terapéuticos no es constante durante el tratamiento, requiriéndose de una administración frecuente de la dosis.

El objetivo de los sistemas de liberación controlada (figura 1) es transportar y dirigir al fármaco al sitio específico del cuerpo donde se necesita, con una rapidez de liberación y una dosis óptima para disminuir los efectos secundarios respecto a la administración convencional y mejorar la biodisponibilidad del fármaco, además de poder ser monitoreados mediante técnicas de fluorescencia, resonancia magnética nuclear o con tomografía (Li, Sheng et al., 2018).

De este modo, los nanomateriales empleados como vehículo transportador de fármacos deben de ser capaces de: i) encapsular una gran cantidad del fármaco (asociado con interacciones de adsorción); ii) controlar la rapidez de liberación del fármaco (relacionado con la degradación hidrolítica del material) y evitar el efecto de explosión (liberación importante en los primeros minutos, que implica altas dosis en periodos cortos de contacto); iii) controlar

Figura 1. Elementos necesarios en la liberación controlada de fármacos.

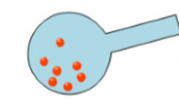

Fármaco
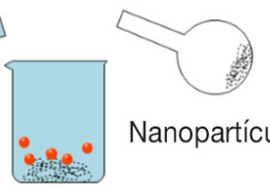

Nanopartículas

Encapsulamiento
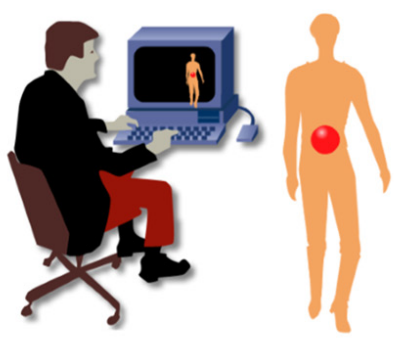

Detección de nanopartículas

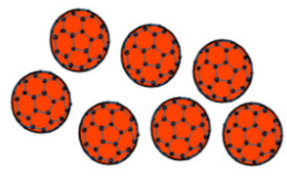

Fármaco encapsulado

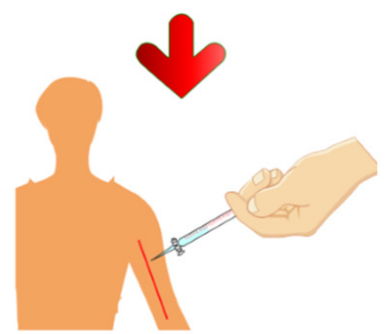

Liberación del fármaco

Fuente: Elaboración de los autores. 
la degradación de la matriz que contiene el fármaco (vinculado con la composición química del material); iv) la posibilidad de modificar su superficie con la finalidad de controlar el transporte a su destino en pruebas in vitro e in vivo (Horcajada et al., 2010); v) biodegradabilidad para evitar su acumulación en el organismo; vi) nula toxicidad y fácil excreción de los productos de degradación; vii) ser fácilmente detectado por técnicas de imagen, y, viii) que su síntesis sea económica, verde y escalable (Christodoulou et al., 2020).

\section{Materiales empleados en la liberación controlada de fármacos}

\section{Nanopartículas inorgánicas}

Diversos grupos de investigación han sintetizado nanopartículas (100 nm o menor) de metales nobles (Ag, $\mathrm{Au}, \mathrm{Pd})$, materiales magnéticos $\left(\mathrm{Fe}_{3} \mathrm{O}_{4}, \mathrm{Co}-\right.$ $\mathrm{Fe}_{2} \mathrm{O}_{4}, \mathrm{FePt}$ ) y semiconductores ( $\mathrm{CdS}, \mathrm{CdSe}, \mathrm{ZnS}$, InP, $\mathrm{PbS}, \mathrm{Si}, \mathrm{TiO}_{2}$ ) (Kaur et al., 2019). Para mejorar la solubilidad y biocompatibilidad de las nanopartículas inorgánicas en el medio fisiológico (figura 2) es necesario su funcionalización con grupos como $-\mathrm{OH},-\mathrm{NH}_{2}, \mathrm{y}-\mathrm{COOH}$ que permiten el acoplamiento con macromoléculas para su uso como vehículo liberador de fármacos. Además, las nanopartículas inorgánicas con fármacos adsorbidos pueden mostrar mecanismos de liberación dependientes de estímulos físicos como el pH, campos eléctricos o magnéticos y radiación electromagnética. Dentro de esta estrategia, nanopartículas de oro pueden liberar moléculas terapéuticas de forma controlada como respuesta a un estímulo de radiación de infrarrojo cercano (Paul y Sharma, 2010).

\section{Nanopartículas magnéticas}

Las nanopartículas magnéticas presentan una alta área superficial, comportamiento paramagnético, baja toxicidad, biocompatibilidad y biodegradación (figura 2). Se ha preferido el uso de nanopartículas de óxido de hierro, en vez de materiales magnéticos como $\mathrm{Fe}$, $\mathrm{Co}$ y $\mathrm{Ni}$, como nanotransportadores en aplicaciones como separación celular in vitro, liberación de genes y liberación controlada de fármacos. Esto se debe a que las nanopartículas magnéticas de hierro son capaces de liberar el fármaco de forma dirigida, lo cual depende de su estructura química rica en grupos - $\mathrm{OH}$ que permiten interacciones de fisisorción con los agentes biológicos encapsulados, las cuales pueden ser modificadas al entrar en contacto con fluidos corporales $(\mathrm{pH}$ y la fuerza iónica) (Amini-Fazl et al., 2019).

Las nanopartículas magnéticas son vehículos que presentan perfiles de liberación dependientes de la intensidad de un campo magnético, lo cual puede ser aprovechado para alcanzar el área afectada; su aplicación se limita a tejidos más externos debido la movilidad limitada en tejidos superficiales (Anirudhan y Christa, 2020; Forouzandehdel et al., 2020). Sin embargo, en aplicaciones en modelos in vivo, su falta de estabilidad coloidal causa su fácil 
remoción del flujo sanguíneo mucho antes de realizar la liberación dirigida del fármaco. Para contrarrestar este problema se han utilizado polímeros como recubrimiento y así obtener una dispersión acuosa estable de nanopartículas de óxido de hierro, mejorando considerablemente la compatibilidad sanguínea y liberación dirigida del fármaco (Amini-Fazl et al., 2019).

\section{Nanopartículas de sílice mesoporosa}

Las nanopartículas de sílice mesoporosa son materiales con alta área superficial (mayor a $500 \mathrm{~m} 2 / \mathrm{g}$ ) y un volumen de poro de aproximadamente $1 \mathrm{~cm}^{3} / \mathrm{g}$ (figura 2). Dichas características permiten la adsorción de moléculas del fármaco; las cuales son adsorbidas en forma de una estructura amorfa incrementando significativamente su capacidad de liberación y biodisponibilidad. La degradación de la silice mesoporosa involucra la hidrólisis ácida o alcalina generando siloxanos ( $\mathrm{Si}-\mathrm{O}-\mathrm{Si}$ ) de bajo peso molecular que no presentan carácter citotóxico a las células. Sin embargo, debe regularse el tamaño de sus partículas para regular su toxicidad y el efecto de explosión (Qian y Bogner, 2012; Bigham et al., 2016).

Por otro lado, la funcionalización de sílice mesoporosa (SBA-15 y MCM41) con grupos amino $\left(-\mathrm{NH}_{2}\right)$ permite una significativa mejora en la capacidad de almacenamiento del fármaco como consecuencia en el incremento de la interacción anfitrión-huésped (Gaudin et al., 2012). Estas nanopartículas de sílice son sensibles a estímulos externos (luz, electricidad) o químicos (cambios de $\mathrm{pH}$ ) haciéndolos materiales útiles para la liberación controlada de fármacos (Angelos et al., 2008; Yao et al., 2018; Zheng et al., 2020).

\section{Nanotubos de carbono}

Los nanotubos de carbono son nanomateriales de grafito huecos y ordenados, con una alta área superficial y con diámetro de 1 a $100 \mathrm{~nm}$. Sus propiedades de semiconducción, estructura unidimensional y alta área superficial, los convierte en materiales con potencial aplicación en biomedicina (Li et al., 2018). Pero antes, deben someterse a un tratamiento de purificación para eliminar restos de grafito, metales ( $\mathrm{Fe}, \mathrm{Mo}, \mathrm{Co}, \mathrm{Ni}, \mathrm{Y}$ ) del catalizador para la síntesis de los mismos, carbón amorfo y pequeños fullerenos; para ello existen tratamientos magnéticos con $\mathrm{HNO}_{3}$.

Los nanotubos de carbono tienen una superficie altamente hidrofóbica y no son solubles en medios acuosos; siendo conveniente su funcionalización mediante el método covalente (formación de un enlace químico) y un enlace no covalente (adsorción física).

La funcionalización por enlace no covalente es la más ampliamente utilizada para su aplicación en liberación controlada de fármacos. La funcionalización es efectuada con moléculas de surfactante (dodecil sulfato de sodio (SDS), dodecil bencensulfonato de sodio (SDBS)) o polímeros anfifílicos (polietilenglicol) o biopolímeros (quitosano, oligonucleótidos, polipéptidos y lisofosfolípidos) preservándose las propiedades físicas de los nanotubos de 
carbono. Debido a su tamaño, los nanotubos de carbono son capaces de cruzar diferentes barreras biológicas, facilitando el transporte y liberación de los fármacos (Li et al., 2017; Maleki et al., 2020; Kaur et al., 2019; Raphey et al., 2019). No obstante, es importante un conocimiento más profundo sobre los mecanismos fisiológicos que provocan la administración de nanotubos de carbono (Sharma et al., 2019).

\section{Liposomas}

Los liposomas son vesículas concéntricas de bicapas de lípidos compuestas de fosfolípidos y colesterol (figura 2). Los liposomas unilaminares y multilaminares son candidatos como vehículos para el transporte de fármacos para enfermedades como cáncer, infecciones fúngicas y degeneración muscular, debido a su alta biocompatibilidad al parecerse estructuralmente a las biomembranas, y su gran capacidad de atrapamiento de especies hidrofílicas e hidrofóbicas son aprovechadas en estrategias para liberación controlada. Típicamente los liposomas son sintetizados por secado en frío, desafortunadamente esta técnica es poco eficiente y consume mucho tiempo y dinero (Wang et al., 2017; Bruch et al., 2019).

Su uso como agente de liberación oral está limitado debido a su inestabilidad en el tracto gastrointestinal y en las dificultades para cruzar la membrana celular (internalización). Para mejorar la estabilidad y la permeabilidad de los liposomas puede modularse la composición de las bicapas de lípidos por la adición de polímeros, proteínas y quitosano (Parker et al., 2019; He et al., 2019). La introducción de liposomas vía parenteral no es práctica, pues son fácilmente eliminados del torrente sanguíneo y terminan en órganos y tejidos del sistema retículo-endotelial, nuevamente se sugiere su recubrimiento con polímeros (polietilenglicol) para evadir el sistema retículo-endotelial y prolongar así su tiempo de residencia en el sistema circulatorio (Lu JuYen et al., 2019; Bunker et al., 2016).

\section{Dendrímeros}

Son una familia de polímeros sintéticos tridimensionales, altamente ramificados y con una estructura bien definida (figura 2). El término dendrímero viene del griego dendron que hace referencia a su estructura ramificada como la de un árbol (Guo y Jiang, 2020; Sherje et al., 2018).

Un dendrímero es típicamente formado por un núcleo con dos o más grupos reactivos y capas internas repetidas unidas covalentemente al núcleo. Debido a sus cavidades internas y la presencia de grupos funcionales terminales, los dendrímeros son considerados vehículos prometedores para el transporte de fármacos. En la actualidad, existen sistemas de liberación de fármacos comerciales basados en dendrímeros de poli(amido-amina), poli(propilen-imina) y poli(L-lisina). Su principal inconveniente es que no se degradan en un ambiente fisiológico, lo cual causa efectos adversos conduciendo a su acumulación dentro de células y tejidos. Una forma de lograr su degradabilidad ha sido el entrecruzamiento 
con ligandos biodegradables como poliésteres, poliacetales y bioproteínas (Huang y Wu, 2018).

\section{Nanopartículas de biopolímeros}

Los biopolímeros (figura 2) más utilizados por su biodegradabilidad, bicompatibilidad y facilidad de procesamiento son los polímeros de ácido láctico, ácido glicólico (PLGA) y sus copolímeros (Zhang et al., 2013). Un polímero biodegradable a diferencia de un polímero sintético no debe provocar un efecto inflamatorio y no debe producir sustancias tóxicas durante su degradabilidad; además, es abundante en la naturaleza y tiene bajo costo. Entre los biopolímeros degradables se tienen los polisacáridos, lo cuales están unidos por un enlace glucosídico, se caracterizan por su alta estabilidad, biocompatibilidad, biodegradabilidad y su fácil modificación. En esta familia se encuentran el ácido hialurónico, quitosano, dextrina, ciclodextrina, polulano y alginato. Otros biopolímeros naturales incluyen polímeros de proteínas como gelatina, colágeno y lecitinas (Duan et al., 2019; Guo y Jiang, 2020; Rajkumar et al., 2019; Prajapati et al., 2019; Wei et al., 2020).

Las técnicas empleadas para la obtención de nanoesferas de polímeros de sacáridos o proteínas son secado en aerosol (bajo rendimiento), extrusión y el método de emulsificación/gelificación; estas dos últimas parecen ser métodos potenciales para la encapsulación de fármacos. El mecanismo de liberación de fármacos empleando nanoesferas de biopolímeros puede dividirse en dos posibles rutas: i) la liberación del fármaco vía la degradación de la estructura del biopolímero, y, ii) la difusión del fármaco a través de la estructura del biopolímero; este último es el que se desea para asegurar la liberación controlada del fármaco (Nguyen Thi Thanh Uyen et al., 2020).

\section{Estructuras metal-orgánicas (MOFs) nanoestructuradas}

Los MOFs pueden ser clasificados con base en su estructura en: i) MOFs de primera generación, son MOFs normales con la estructura básica del polímero de coordinación; ii) MOFs de segunda generación, que corresponden a MOFs funcionalizados o recubiertos, y, iii) MOFs de tercera generación, también llamados MOFs inteligentes compuestos de cationes biocompatibles, capaces de incorporar dentro de su estructura o en su superficie fármacos y moléculas bioactivas (Beg et al., 2017). Dentro de estos tipos de MOFs, los de gran interés de aplicación biomédicas son los de segunda y tercera generación; ya que pueden emplearse en: i) la encapsulación, protección y liberación de fármacos; ii) imagen biomédica para el rastreo y distribución en tiempo real del fármaco, y, iii) como agentes terapeúticos, especialmente en el tratamiento contra el cáncer (Banerjee et al., 2020).

Los MOFs nanoestructurados para la liberación de fármacos son el resultado del ensamble de clusters metálicos y ligandos orgánicos con grupos carboxílicos, fosfatos e imidazolatos; los cuales son grupos reactivos para la 
formación de nuevas funcionalidades (figura 2). Así, la estructura química del nanoMOF presenta regiones hidrofílicas e hidrofóbicas que modulan su compatibilidad sanguínea. Además, su alta área superficial implica mayores sitios de adsorción para las moléculas terapéuticas y el mecanismo de liberación puede ser influenciado por la estructura química del ligando orgánico (Zhang et al., 2018; Abanades y Forgan, 2019). La presencia de un catión metálico específico influye en la porosidad del material nanoestructurado y en la modulación de la respuesta biológica de las células implicadas en el proceso de curación (Horcajada et al., 2010).

Los nanoMOFs son bioplataformas para la aplicación de terapias combinadas: 1) la encapsulación de dos o más ingredientes activos (fármacos, biomoléculas, gases, metales) simultáneamente o secuencialmente; 2) la liberación controlada en un sitio específico mediante un estímulo externo (luz infrarroja, campo magnético); 3) el monitoreo de la administración a través de bioimagen; 4) la combinación de la liberación y diagnóstico (teragnosis), y, 5) la detección de diferentes analitos empleando biosensores (Vahed et al., 2019; Zhang et al., 2019).

\section{Biocompatibilidad de los nanoMOFs}

La biocompatibilidad de los MOFs depende de su composición química: el ión metálico, el ligando orgánico e incluso el solvente empleado en su síntesis. Se recomienda la síntesis de nanoMOFs empleando iones metálicos bio-

Figura 2. Características principales de los materiales para liberación controlada de fármacos.

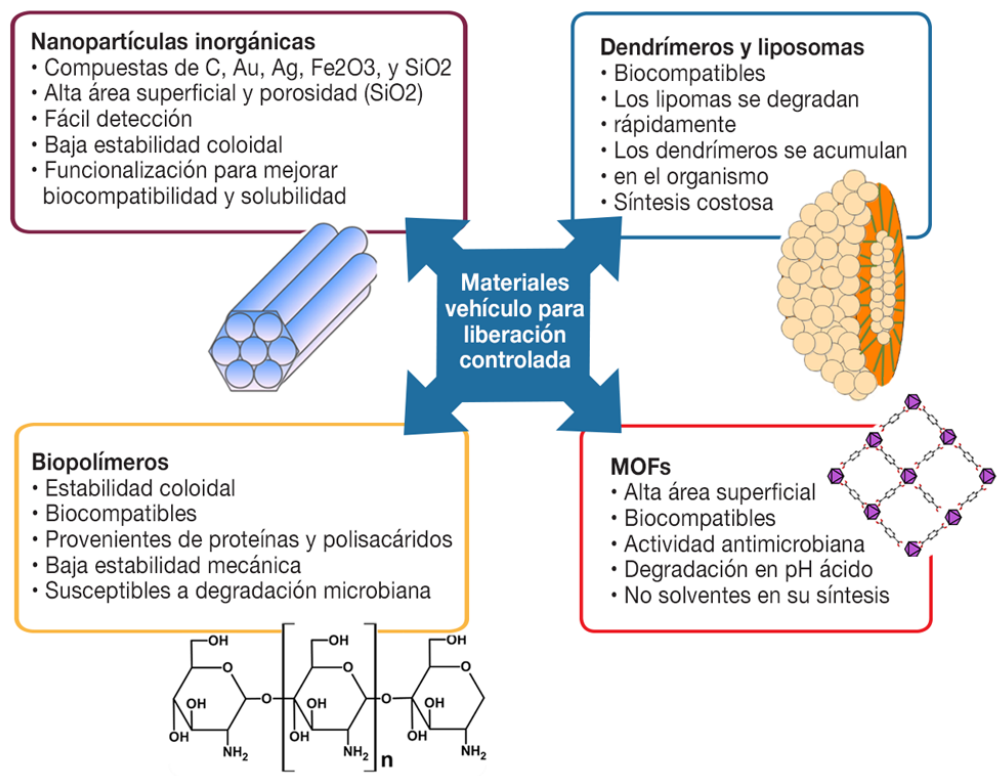

Fuente: Elaboración de los autores. 
compatibles como $\mathrm{Mg}^{+2}, \mathrm{Ca}^{+2}, \mathrm{Fe}^{+3}, \mathrm{Fe}^{+2}$ y $\mathrm{Zn}^{+2}$. Adicionalmente, existen metales encontrados en trazas en el cuerpo humanos que son esenciales $(\mathrm{Cu}$, $\mathrm{Mn}, \mathrm{Se}, \mathrm{Mo}, \mathrm{Cr}, \mathrm{V}$ ) actuando como cofactores de enzimas o como sitios activos para ellas, así como metales inertes ( $\mathrm{Au}, \mathrm{Ag}, \mathrm{Zr}$ ) que pueden ser utilizados (Horcajada et al., 2010; Rojas et al., 2019).

Dentro de los ligandos empleados se tiene a fosfonatos, carboxilatos, imidazolatos y aminas, los cuales causan irritación en el tracto respiratorio (boca, nariz), ojos y piel (Mohammed et al., 2019). No obstante, los ligandos orgánicos con grupos carboxílicos e imidazolatos, debido a su gran polaridad pueden removerse fácilmente de los animales mediante la orina y heces (Christodoulou et al., 2020; Pandey et al., 2020). Por otro lado, el ligando orgánico también puede ser una molécula endógena del organismo como los son los aminoácidos, péptidos, proteínas, bases nucleicas, carbohidratos, fosforina o moléculas exógenas como ácido nicotínico, curcumina y la olsalazina (Rojas et al 2019). En relación con el solvente, la preparación del MOF implica solventes como etanol, cloroformo, dimetilsulfóxido (DMSO) y dimetilformamida (DMF), cuyos residuos en el MOF pueden causar toxicidad renal y hepática; siendo recomendable solo el uso de agua o etanol como solvente (Lakshmi y Kim, 2019; Mohammed et al., 2019). De esta manera, los MOFs más explotados en la liberación de fármacos comprenden los MOF de las familias ZIF-8, UiO-66 y MIL (MIL-53, MIL-88, MIL-89, MIL-100 y MIL101) (Christodoulou et al., 2020).

\section{ZIF (Zeolitic imidazolate frameworks)}

Son una clase de MOFs no tóxicos basados en $\mathrm{Zn}^{2+}$ con el 2-metilimidazol y el 3-metilimidazol aplicados en biomedicina (figura 3). Los MOFs del tipo ZIF-8 han sido los más estudiados para la liberación de fármacos debido a su especial estabilidad termoquímica, alta capacidad de almacenamiento, modulación de sus sitios funcionales, un gran tamaño de poro, habilidad de adsorción y liberación de fármacos.

El ZIF-8 tiene ventajas únicas como vehículo de macromoléculas debido a: i) sus interacciones hidrofóbica y por puente de hidrógeno entre la molécula huésped y el grupo imidazol (fisisorción incrementada), facilitando el almacenamiento de la misma; ii) se prepara obteniendo el tamaño de la partícula en escala nanométrica, siendo útil para la liberación de fármacos dirigida a nivel celular; iii) los iones de zinc se disocian del ligando imidazol en un rango de $\mathrm{pH}$ de 5-6 debido a su protonación, es decir, permite la liberación controlada de la macromolécula dentro del citoplasma celular, exhibiendo un perfil de liberación controlada (Banerjee et al., 2020; Zhong et al., 2019). Aunque, su rápida degradación en un rango de $\mathrm{pH}$ ácido, que genera que una cierta cantidad de iones zinc, sea también lixiviada del MOF, resultando en la generación de especies de oxígeno reactivas y por consecuencia en la muerte de células normales (Vahed et al., 2019). 


\section{Uio (Universitet i Oslo)}

La familia de MOFs UiO son clusters de estructuras octaédricas del tipo $\left[\mathrm{Zr}_{6} \mathrm{O}_{4}(\mathrm{OH})_{4}(\mathrm{RCOO})_{12}\right]$ unidas a ligandos orgánicos de dicarboxilatos lineales (figura 3) dentro de una estructura altamente porosa con fórmula $\left[\mathrm{Zr}_{6} \mathrm{O}_{4}(\mathrm{OH})_{4}\right.$ $\left.(\mathrm{L})_{6}\right]_{\mathrm{n}}$. La primera serie de MOFs de la familia UiO se realizó utilizando como ligando al ácido tereftálico (BDC, 1,4-Bencendicarboxilato) mostró un área de $1200 \mathrm{~m}^{2} / \mathrm{g}$ y un volumen de poro de $0.5 \mathrm{~cm}^{3} / \mathrm{g}$. El uso de ligandos que contienen grupos carboxilatos permite la modificación post-sintética de los MOFs, ya que los grupos carboxilatos de la superficie pueden ser sustituidos por otros (intercambio de ligando) o en su defecto realizar reacciones de conjugación usando los grupos carboxilatos obteniendo diversas funcionalidades (Shijun Tai et al., 2016; Pander et al., 2018; Abanades y Forgan, 2019). Recientemente, se ha incrementado el interés en MOFs a base de $\mathrm{Zr}^{4+}$, debido a la baja toxicidad del zirconio y al relativo tamaño amplio de sus poros (Zhang et al., 2020). La fuerte naturaleza ácido-base de los enlaces Zr-grupos carboxílicos, los convierte en un material con mejores propiedades químicas y mecánicas que un MOF a base de hierro u otro de valencia alta; lo cual facilita su posterior funcionalización sin comprometer su estructura química, porosidad y capacidad de almacenamiento, permitiendo modular la biodegradación y, por lo tanto, presentar perfiles de liberación controlada (Pander et al., 2018; Liu et al., 2019).

\section{MIL (Materials of Institute Lavoisier)}

La familia MIL (53, 88, 100 y 101) está formada por centros metálicos trivalentes y ligandos compuestos de ácidos carboxílicos, posee poros hidrofóbicos de 25-34 $\AA$, una superficie de 3100-5900 m²/g; siendo ideal para el encapsulamiento de fármacos poco solubles en agua (Huxford et al., 2010). Típicamente se han empleado centros metálicos como el $\mathrm{Al}^{3+}$ y el Fe${ }^{3+}$, este último debido a su alta biocompatibilidad (figura 3). Más aun, la funcionalidad de los grupos carboxílicos de sus ligandos orgánicos ha sido modificada con grupos amino, haciendo posible el acomodo de macromoléculas en sus cavidades, por interacciones de fisisorción. Estos grupos amino son susceptibles a reaccionar a cambios de $\mathrm{pH}$, lo cual puede estimular la liberación del fármaco luego de atravesar la membrana celular y mejorar la liberación sostenible del fármaco (Cabrera-García et al., 2019).

\section{Estructuras metal-orgánicas biodegradables (BioMOFs)}

Para resolver el inconveniente de la toxicidad del ligando, se han sintetizado bioMOFs a base de ligandos biodegradables endógenos como lo son enzimas, anticuerpos, bases nucleicas, péptidos, proteínas y porfirinas, así como ligandos biodegradables exógenos como la ciclodextrina, fumarato, galactorato y el ácido azelaico (Beg et al., 2017; Cai et al., 2019; Christodoulou et al., 2020; Lakshmi y Kim, 2019).

Así, las bases nucleicas como la adenina y purina son las moléculas más ampliamente estudiadas para la construcción de bioMOFs debido a su bajo 
Figura 3. Familias de MOFs empleadas en la liberación controlada de fármacos.

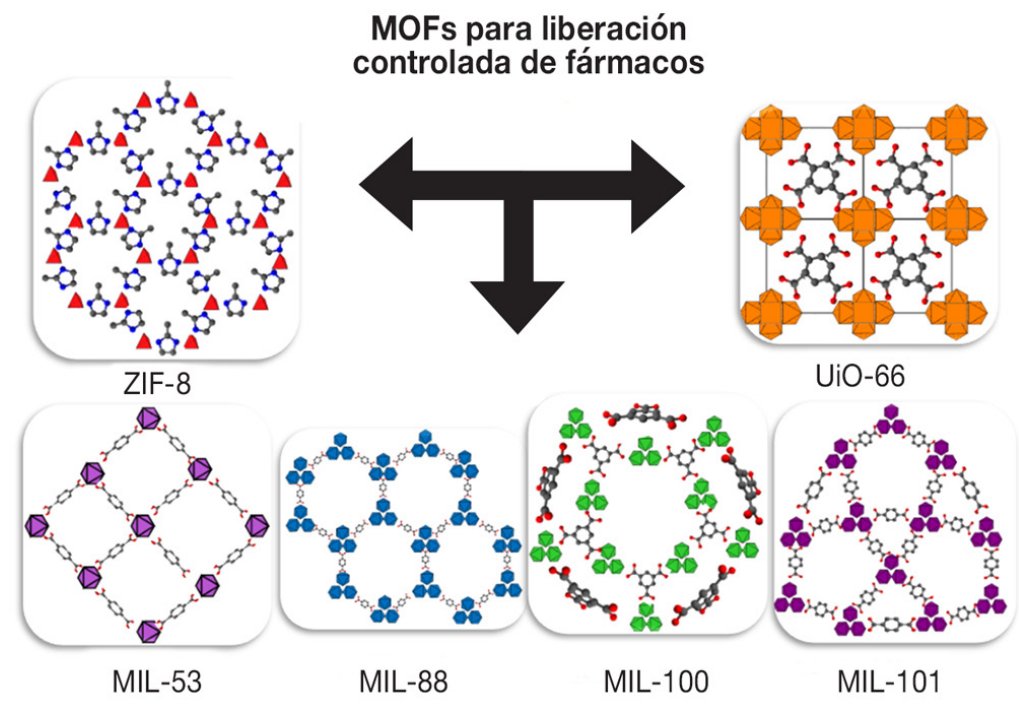

Fuente: Elaboración de los autores.

costo y fácil preparación; además de contener varios átomos accesibles de nitrógeno y oxígeno con pares electrónicos libres. A su vez, los aminoácidos sirven como ligandos orgánicos debido a la presencia de sus grupos amino y carboxílico (An et al., 2019; Cai et al., 2019). Estos bioMOFs sintetizados inicialmente para la liberación de fármacos pueden ser empleados en biocatálisis, biosensores, celdas de biocombustibles y aparatos bioelectrónicos (An et al., 2019). Algunas de las desventajas de estos bioMOFs son que se obtienen estructuras con interacciones muy lábiles entre metal y ligando facilitando la interpenetración, obteniendo geometrías no favorables que resultan en estructuras no porosas.

\section{Funcionalización de MOFS}

La funcionalización de MOF con grupos funcionales polares $\left(-\mathrm{NH}_{2},-\mathrm{OH}\right) \mathrm{o}$ donadores de protones han demostrado la mejora en el encapsulamiento de macromoléculas por fisisorción debido a los puentes de hidrógeno generados entre ellas y el MOF. Mientras que en ausencia de estos grupos, la capacidad de almacenamiento puede ser mejorada por el incremento de las fuerzas de Van der Waals en su superficie mediante la adición de mono, di y tetrasustituidos grupos metilo, aprovechado para fármacos de naturaleza hidrofóbica (Gaudin et al., 2012).

Para la funcionalización de MOFs para su aplicación en la liberación de fármacos, se emplean ligandos orgánicos con grupos funcionales nucleófilos 
como carboxilatos, tioles, fosfatos e imidazolatos para facilitar su funcionalización in situ en la síntesis del MOF o post-síntesis.

\section{Funcionalización post-síntesis}

La funcionalización post-síntesis puede dividirse en: i) coordinativa; ii) covalente; iii) no covalente, y, iv) modificación externa del MOF.

En la funcionalización post-síntesis coordinativa, se realiza un intercambio del ligando orgánico. Este proceso se puede efectuar también en el proceso de síntesis utilizando un ligando monodentado (modulador) que contenga grupos $\mathrm{COOH}-$, el cual se añade a la mezcla de reacción que contiene ligandos polidentados. De esta forma, la adición de un ligando monodentado puede regular la coordinación debido a una competición con el ligando polidentado para coordinarse con el ión metálico. De esta forma, la relación de concentraciones del ligando modulador y del ligando incorporado regula y controla el proceso de nucleación de los cristales del MOF (Khan et al., 2018; Pandey et al., 2020). En la modificación post-sintética se utilizan ligandos con grupos nucleófilos que incluyen fosfatos, tioles, carboxilatos e imidazoles para modificar la superficie del MOF; así, las biomoléculas que se quiere incorporar remplazan a los ligandos de la superficie (Zhang et al., 2020).

En la funcionalización covalente, el ligando sirve como plataforma para reacciones químicas posteriores para cambiar los grupos funcionales terminales del ligando orgánico o incrementar el tamaño de su cadena hidrocarbonada. Por ejemplo, se puede dar la conjugación de péptidos a los grupos amino libres del ligando del MOF MIL-68- $\mathrm{NH}_{2}$. Además, los grupos nucleófilos de las proteínas como los grupos $-\mathrm{NH}_{2}, \mathrm{OH}^{-}$y $\mathrm{SH}^{-}$permiten su conjugación covalente con el ligando del MOF mediante sustitución nucleófílica (Zhang et al., 2020). Estas estrategias pueden ser aprovechadas para mejorar tanto la solubilidad, compatibilidad sanguínea, fisisorción y degradación del MOF para aplicaciones biomédicas (Pandey et al., 2020).

La modificación post-sintética no covalente se utiliza principalmente para la modificación de la superficie empleando macromoléculas como proteínas lo cual involucra interacciones electrostáticas, puentes de hidrógeno y fuerzas de dispersión (Pandey et al., 2020; Zhang et al., 2020).

La modificación de la naturaleza de la superficie del MOF permite su adecuada interacción con el medio fisiológico (solvente, proteínas, lípidos, iones, enzimas, etc.). Por ejemplo, el reconocimiento y la adherencia de proteínas a la superficie de los nanoMOFs, proceso conocido como opsonización, puede afectar su distribución dentro del cuerpo, lo cual resulta en su eliminación mediante el sistema reticuloendotelial (RES) y, finalmente, del cuerpo. Así, para ganar propiedades de bioadherencia de los MOFs, su superficie externa puede ser revestida con sílice, polivinil pirrolidona, polisacáridos como el dextrano, biotina, ciclodextrina o quitosano, liposomas, heparina, fluoresceína o ácido fólico (Rojas et al., 2019; Zhang et al., 2020). 
Para lograr la adherencia de los polímeros a las partículas de nanoMOFs es necesario que los polímeros contengan grupos funcionales de: i) naturaleza iónica como fosfatos, sulfatos y carboxilatos, para lograr una fuerte interacción, o, ii) mediante interacciones débiles (puente de hidrógeno, fuerzas de Van der Waals) para lograr un efecto cooperativo fuerte. Por caso, la ciclodextrina puede ser modificada con iones fosfato para adherirse a nanopartículas de MOFs; de este modo, la ciclodextrina interacciona primero electrostáticamente con la superficie del MOF, y luego los iones fosfato son coordinados selectivamente con los sitios ácidos de Lewis (centros metálicos coordinativamente insaturados, CUS) de la superficie del MOF. Otra forma de adherencia de los polímeros es mediante enlace covalente de los grupos reactivos del polímero con los ligandos formando una capa protectora, este tipo de recubrimiento se puede realizar durante la síntesis o post-síntesis (Giménez-Marqués et al., 2016; Beg et al., 2017).

\section{Síntesis de MOFs nanoestructurados}

\section{Método solvotermal/hidrotérmico}

Involucra la disolución de los precursores metálicos en solventes polares y de los precursores inorgánicos en solventes orgánicos bajo condiciones moderadas de temperatura $\left(80-180^{\circ} \mathrm{C}\right)$ y presión con tiempos de reacción en un rango de 21-96 horas (Bashir et al., 2020) (figura 4). Algunas familias de MOFs sintetizadas mediante este método son las del tipo MIL-88A, MIL88B, MIL-89, MOF-5, UiO-66 y ZIF-8. Los parámetros a controlar son la temperatura, el tiempo de reacción, el pH y la relación molar metal/ligando orgánico (Giménez-Marqués et al., 2016).

\section{Sintesis asistida por microondas}

Esta técnica es una alternativa al calentamiento tradicional, permitiendo la rápida cristalización de los MOFs (figura 4). Especialmente usando microondas favoreciendo la formación de puntos calientes $\left(30-100^{\circ} \mathrm{C}\right)$, y una rápida transferencia de calor obteniendo partículas con tamaño de partícula homogéneo en tiempos de 0.1 a 4 horas (Bashir et al., 2020; Giménez-Marqués et al., 2016). Sin embargo, se requiere encontrar el método adecuado para la evaporación del solvente, incluyendo el desarrollo de cristales en soluciones saturadas y mejorar la solubilidad con la temperatura, obteniendo así los cristales durante la fase de enfriamiento (Beg et al., 2017; Khan et al., 2018; Safaei et al., 2019). Dada la temperatura alcanzada por este método, este no es útil para emplear proteínas como ligando (Pandey et al., 2020).

\section{Método sonoquímico}

Uso de energía alta de ultrasonido $(10 \mathrm{MHz})$ a una mezcla de reacción a temperatura ambiente (una temperatura máxima de $40^{\circ} \mathrm{C}$ ) lográndose la dispersión de pequeños aglomerados de partículas (figura 4). Las reacciones quími- 
cas se efectúan en el líquido que presenta cavitación en la interfase o en el medio donde existen fuerzas de cizallamiento, incluso puede darse la formación de radicales (Safaei et al., 2019). No es recomendable usar mucho tiempo de ultrasonido ( 0.5 a 3 horas) pues se incrementa el tamaño de partícula y el MOF comienza a degradarse (Pandey et al., 2020).

\section{Síntesis mecanoquímica}

Esta síntesis se realiza mediante la molienda de la mezcla de las sales de los metales de los precursores o sus óxidos metálicos junto con las sales de ligando inorgánico (figura 4), obteniéndose solo agua como subproducto (Pandey et al., 2020). Las ventajas de esta síntesis es que no requiere de ningún solvente para su preparación y se obtienen altos rendimientos en tiempos cortos de reacción (0.5 a 2 horas) (Bashir et al., 2020; Safaei et al., 2019). Pero parece estar limitada a MOFs cuya síntesis se realiza bajo condiciones suaves de reacción, excluyendo MOFs de metales como $\mathrm{Fe}^{2+}, \mathrm{Al}^{3+}, \mathrm{Cr}^{3+}, \mathrm{Zr}^{4+} \mathrm{o} \mathrm{Ti}^{4+}$; los cuales solo pueden obtenerse mediante un método solvotermal o hidrotérmico (Giménez-Marqués et al., 2016; Nadizadeh et al., 2018).

\section{Síntesis por microemulsión}

Este procedimiento involucra una dispersión termodinámicamente estable de dos líquidos inmiscibles en presencia de un emulsificante o agente surfactante (figura 4). La morfología y tamaño de la partícula quedan restringidas a la relación agua/surfactante. Este procedimiento también ha sido aplicado en la metodología sol-gel para modular la morfología y tamaños de las nanopartículas (Giménez-Marqués et al., 2016; Ren et al., 2017).

\section{Método electroquímico}

Los MOFs en polvo se producen a escala industrial con este método, se evita el uso de sales metálicas y se alcanza una temperatura máxima de $30{ }^{\circ} \mathrm{C}$ y tiempos de reacción entre 10 a 30 minutos (Bashir et al., 2020; Safaei et al., 2019). El ión metálico se obtiene a través del ánodo mientras que el ligando se disuelve en la mezcla de reacción junto con el electrolito (figura 4). La deposición del ión metálico desde el ánodo al cátodo es suprimido por el uso de solventes próticos en la mezcla de reacción. Esta metodología sirve para el recubrimiento de MOFs y la formación de películas de MOFs (Lakshmi y Kim., 2019; Pandey et al., 2020).

\section{Producción a escala industrial}

Solo algunos MOFs con tamaño de partícula en la escala micrométrica han sido sintetizados a escala industrial entre ellos Basolite A100 (MIL-53(Al)), Basolite C300 (HKUST-1), Basolite F300 (FeBTC), Basolite Z1200 (ZIF-8) y Basosive M050 (Mg formate). Se prefieren los procesos de flujo continuo para la producción a escala industrial, este se efectúa utilizando la síntesis por el método solvotermal utilizando un reactor continuo (figura 4), con 
Figura 4. Metodologías de síntesis de MOFs nanoestructurados.

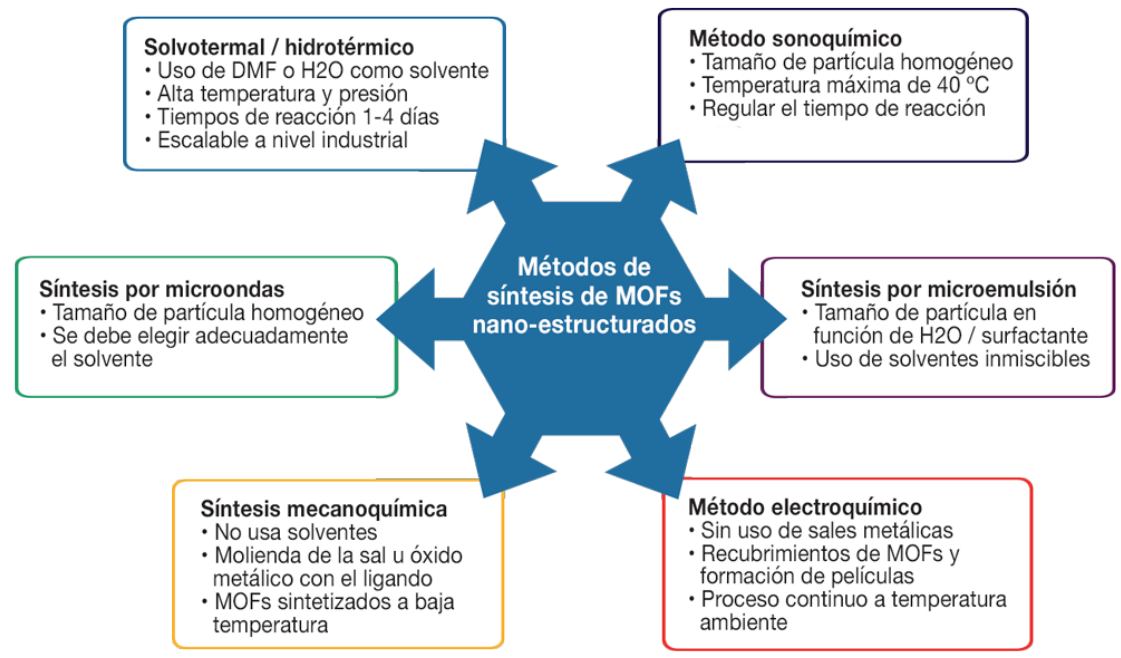

Fuente: Elaboración de los autores.

base en el reactor desarrollado en 1990 para la fabricación industrial de zeolitas (Evans et al., 2019). En este caso, el factor más importante para la síntesis de los MOFs es el solvente empleado, delimitando la temperatura de reacción, presión de operación y la concentración de la mezcla de reacción (Shijun Tai et al., 2016).

\section{Métodos de encapsulamiento en MOFS}

\section{Impregnación}

Es uno de los métodos más comunes para la encapsulación de fármacos o moléculas bioactivas. En esta técnica las moléculas que se deben encapsular, se disuelven en soluciones acuosas o etanólicas, proceso en el cual las moléculas del fármaco se incorporan dentro de los poros del MOFs por difusión, mediante interacciones no covalentes con los sitios metálicos o el ligando. (Christodoulou et al., 2020; Zhang et al., 2020).

\section{Encapsulación in-situ}

Cuando el tamaño de las moléculas a encapsular es mayor al tamaño de los poros del nanMOFs, la encapsulación in-situ resulta una buena opción, por ejemplo, las proteínas. Para ello, se dispersan proteínas dentro de la solución donde son sintetizadas las partículas de MOFs, eventualmente, las partículas de nanoMOFs se construyen alrededor de las proteínas. En este caso, las interacciones hidrofóbicas y los puentes de hidrógeno entre las proteínas y los agregados metálicos son la base para el éxito de la encapsulación (Zhang et al., 2020; Gulcay y Erucar, 2020). 


\section{Encapsulación de moléculas bioactivas}

La encapsulación de biomoléculas como proteínas para terapia de remplazo de proteínas y enzimas de ADN para terapia genética pueden ser incorporadas en sistemas de liberación de estímulo-respuesta (MOFs inteligentes), siendo preferible que las unidades estructurales de los MOFs en sí no sean tóxicos. Un ejemplo es el uso del MOF UiO-66 con alta estabilidad debido a su composición de carboxilatos de zirconio, el cual puede modular la liberación de su carga respecto a cambios de $\mathrm{pH}$, temperatura o incluso concentración de iones para impedir o permitir la liberación de las biomoléculas que transporta (Banerjee et al., 2020; Xu y Li., 2020).

\section{Encapsulación dentro de los poros del MOF}

Se puede modular el tamaño de poro de los MOFs obteniendo materiales ultra-microporosos hasta mesoporosos. Este es el método más directo y más ampliamente usado para la encapsulación de biomoléculas, pues no se compromete la estructura química de la biomolécula (figura 5). Se requiere de MOFs con una buena estabilidad al agua y un amplio tamaño de poro. En este proceso las biomoléculas deben entrar con una diferente estructura conformacional, pudiendo retener su actividad. Los MOFs PCN-332 y PCN-333 han llamado la atención debido a su alta estabilidad en agua y capacidad de atrapar enzimas (Wang et al., 2018; An et al., 2019).

\section{Biomoléculas adheridas a la superficie del MOF}

En este caso, el tamaño de poro del MOF no es una restricción y el proceso de inmovilización es rápido y fácil comparado con otros métodos (figura 5 a). Sin embargo, su principal desventaja es el lixiviado de la biomolécula. Las interacciones por puente de hidrógeno e interacciones $\pi-\pi$ contribuyen principalmente a la atracción anfitrión-huésped entre las biomoléculas y el ligando (An et al., 2019).

\section{Biomoléculas unidas al MOF por enlace covalente}

Para evitar el lixiviado de las moléculas, es necesario crear enlaces covalentes entre la estructura del MOF y las biomoléculas (figura 5b), para lo cual se usan grupos nucleófilos como grupos amino, carboxílicos, fenólicos, tioles, imidazol, indol e hidroxilo en las biomoléculas, las cuales pueden formar interacciones covalentes con el ligando del MOF. Además, la superficie de los cristales del MOF también presenta grupos terminales (carboxílico, amino, hidroxilo) que pueden ser acoplados con biomoléculas. Hasta el momento existen tres métodos (An et al., 2019; Gulcay y Erucar, 2020) para incorporar covalentemente biomoléculas:

a) Las biomoléculas pueden unirse a agentes entrecruzantes como el glutaraldehído formando una monocapa alrededor de la superficie del MOF. 
b) Las biomoléculas pueden acoplarse directamente a los agregados metálicos del MOF por enlaces covalentes.

c) Las biomoléculas pueden previamente ser conjugadas con ligandos orgánicos y luego sintetizar el MOF (An et al., 2019).

\section{Encapsulación in-situ}

En esta técnica los cristales del MOF crecen alrededor de las biomoléculas dando lugar a una alta eficiencia de absorción y casi no se presenta lixiviado (figura 5c). La nucleación e inmovilización ocurren simultáneamente en un solo paso. No obstante, el proceso solo puede efectuarse con soluciones acuosas bajo condiciones suaves de temperatura y evitando el uso de solventes orgánicos (An et al., 2019).

\section{Mecanismo de liberación de fármacos con MOFs}

La liberación de fármacos y agentes biológicos en un sitio específico es una de las aplicaciones más populares de los MOFs inteligentes, capaces de responder a estímulos externos como luz, $\mathrm{pH}$, temperatura, presión u otros campos externos (campo magnético) para regular la liberación de la carga. De esta manera, se puede rastrear en tiempo real la ubicación y distribución del MOF dentro del organismo mediante imagen por resonancia magnética o tomografía por emisión de positrones (Banerjee et al., 2020). La velocidad

Figura 5. Estrategias de encapsulación de moléculas bioactivas.

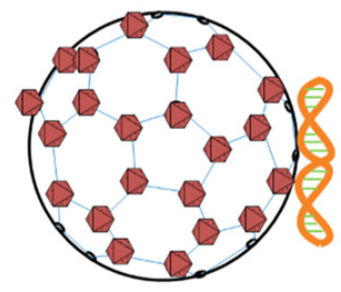

(a) Biomoléculas adheridas a la superficie del MOF

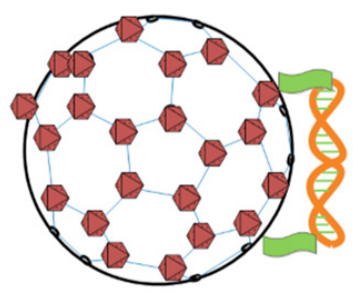

(b) Biomoléculas

unidas por enlace covalente

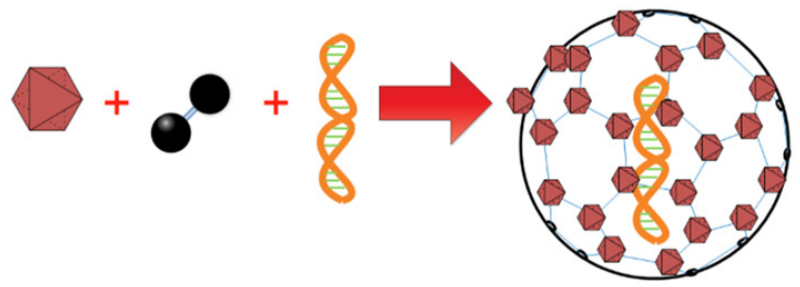

(c) Encapsulación in situ

Fuente: Elaboración de los autores. 
de liberación de cada tipo de carga depende de la interacción de esta con el MOF. Así, una fuerte interacción puede dificultar la liberación, en tanto que una débil interacción entre el huésped y el MOF vuelve ineficiente la encapsulación (Banerjee et al., 2020).

La estructura del MOF debe ser estable durante el proceso de encapsulación, recubrimiento y durante su circulación en el organismo vivo, hasta que llegue al sitio específico. Los MOFs deben ser recubiertos en un cascarón que les permita alcanzar su objetivo biológico y que además sea biodegradable (Christodoulou et al., 2020).

$\mathrm{El} \mathrm{pH}$ del microambiente de un tumor ( $\mathrm{pH}$ 5.7-7.8), endosoma ( $\mathrm{pH}$ 5.56.0) y lisosoma (4.5-5.0) tiende a ser más ácido del $\mathrm{pH}$ encontrado en tejido y sangre sana (7.35-7.45). Muchas de las familias de MOFs (ZIF, MIL y UiO) empleadas como vehículos de fármacos son fácilmente hidrolizadas en medio ácido debido a la labilidad de los enlaces amina, oxima, ester, imina y poliacetal. La hidrólisis ácida de los MOFs genera oligómeros de bajo peso molecular que tienden a liberar el fármaco dentro de la célula, facilitando la llegada a los receptores celulares específicos; por ello y para evitar la muerte celular, estos oligómeros deben poseer baja citotoxicidad.

Por ejemplo, en MOFs a base de $\mathrm{Zr}^{4+}$ esto puede efectuarse mediante el encapsulamiento del fármaco en la superficie y defectos superficiales del MOF mediante enlaces de fisisorción, lo cual hace que el pH del fluido fisiológico o concentración de fosfatos induzcan la liberación del fármaco. Así, la eficiencia terapéutica de cualquier tipo de vehículo transportador de fármaco está limitada a su capacidad de atravesar membranas celulares (parte hidrofóbica del MOF) y liberar el fármaco a los diversos compartimentos de la célula (Abanades y Forgan, 2019).

\section{Protonación inducida del ligando}

En este caso los MOFs se componen de ligandos orgánicos de grupos funcionales ionizables como el imidazol, amina, carboxílico, piridina, imino; los cuales son desprotonados en un medio fisiológico con $\mathrm{pH}=7.4$ (figura 6a). Sin embargo, pueden ser protonados otra vez en un microambiente generado por un tumor, resultando en el rompimiento de los enlaces de coordinación entre el metal y el ligando orgánico y la liberación de carga del fármaco en el sitio del tumor. Las familias de MOFs que resaltan por seguir este mecanismo de liberación son los MOFs tipo ZIF, MIL, UiO y DUT (Wang et al., 2019).

\section{Rompimiento de enlaces sensibles al pH}

Una manera de adherir los fármacos al vehículo liberador es mediante un enlace covalente para tener una alta selectividad hacia un tumor objetivo (figura 6b). La liberación del fármaco se alcanzará mediante la hidrólisis de enlaces a $\mathrm{pH}$ bajo entre el fármaco y el MOF. La rapidez con que el pH puede romper estos enlaces puede ser modulada seleccionando ligandos sensibles al $\mathrm{pH}$ con grupos funcionales como la hidracina, enlace epoxídico, imina y 
amida. En un pH ácido el rompimiento de estos enlaces resulta en la liberación del fármaco. Suelen utilizarse los enlaces tipo amida para la unión entre el fármaco y el MOF; por caso, algunos fármacos anticancerígenos son ricos en grupos amino los cuales se anclan covalentemente con MOFs que contiene grupos carbonilo $(-\mathrm{C}=\mathrm{O})$ (Wang et al., 2019).

\section{Interacción huésped-anfitrión}

Este tipo de interacciones ocurren entre el fármaco y el MOF (figura 6c). Involucra interacciones del tipo electrostáticas, por puente de hidrógeno, interacciones $\pi-\pi$, que al entrar en contacto con un $\mathrm{pH}$ ácido provocan un debilitamiento de estas interacciones y por ende la liberación controlada del fármaco (Wang et al., 2019).

\section{Rompimiento de enlaces de materiales sensibles al pH}

Se utiliza como recubrimiento del MOF un material sensible al $\mathrm{pH}$ como los biopolímeros; el material es degradado, para luego permitir la degradación controlada del MOF y por ende la liberación del fármaco (figura 6d). Por mencionar alguno, en el caso de quitosano con grupos terminales amino $\left(-\mathrm{NH}_{2}\right)$, estos generan especies $\mathrm{NH}^{3+}$ a un $\mathrm{pH}$ menor a 6.3. Lo cual da lugar a una fuerte estructura con puentes de hidrógeno internos, que permiten modular la liberación del fármaco del vehículo. En este tipo de sistemas de liberación controlada, el MOF puede formar el núcleo encapsulando el fármaco o ser parte del recubrimiento del fármaco (Wang et al., 2019).

Figura 6. Estrategias de encapsulación de moléculas bioactivas.

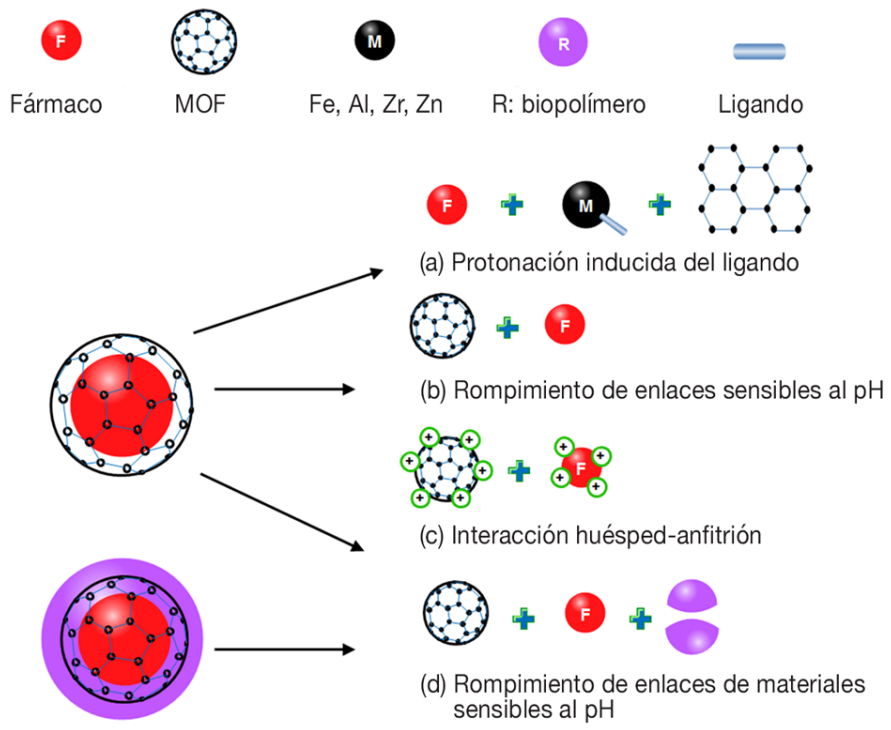

Fuente: Elaboración de los autores. 


\section{MOFs como agentes terapeúticos contra el cáncer}

En el proceso de liberación de fármacos efectivos contra el cáncer, se requiere que el sistema de liberación controlada entre y circule dentro del sistema circulatorio, luego que se acumule en el tumor, que penetre lo más que se pueda dentro del tejido del tumor y que se interne dentro de las células del tumor para permitir la liberación del fármaco (Mohammed et al., 2019)

Los nanoMOFs son materiales prometedores para su uso en el combate contra el cáncer debido a sus características que incluyen: 1) su alta capacidad de ligarse a células cancerígenas; 2) mejora la citotoxicidad del fármaco; 3) los nanoMOFs pueden liberar el fármaco como respuesta a estímulos como el pH y la luz (Dong et al., 2019; Li et al., 2019; Lu et al., 2019; Mohammed et al., 2019; Zhang et al., 2018; Zhu et al., 2019).

Los nanoMOFs tienen una buena interacción con las nanomoléculas prexistentes para el combate de cáncer como liposomas, proteínas, micelas poliméricas con elementos inorgánicos como oro, plata, sílice mesoporosa y magnetita $\left(\mathrm{Fe}_{3} \mathrm{O}_{4}\right)$. Además, su naturaleza con entidades hidrofílicas e hidrofóbicas permite la adsorción de fármacos y su aplicación como agente antibacterial, angiogénesis y terapia genética (Mohammed et al., 2019).

Fármacos como doxorrubicina, 5-fluorouracil, zoledronato cis-platino y metotrexato han sido conjugados éxitosamente a nanoMOFs, además de ser eficaces contra el cáncer debido a la adecuada retención del fármaco y la acumulación del nanoMOF en el tumor objetivo; aunado a su monitoreo en tiempo real con alguna técnica de imagen (Cai et al., 2019; Mohammed et al., 2019; Zhang et al., 2019).

\section{Actividad antimicrobiana de MOFs}

Los MOFs pueden actuar como vehículo transportador de nanopartículas metálicas con propiedades antibactericidas como la plata. Se sabe que las propiedades químicas, el tamaño y forma de la partícula, junto con el potencial zeta; son factores que determinan la actividad antibactericida de los sólidos. Así, las nanopartículas metálicas previenen la formación de agregados de partículas y promueven su interacción con las bacterias pudiendo atravesar sus paredes celulares e inhibir su crecimiento.

La actividad biocida de nanopartículas de metales y óxidos metálicos es atribuida a la formación de especies de oxígeno reactivos mediante un agente externo como la fotoactivación por luz UV. La capacidad bactericida de los MOFs se relaciona con los precursores orgánicos e inorgánicos, los cuales determinan su estructura química. La propiedad antimicrobiana del MOF se asocia con la interacción del MOF con la pared celular bacteriana, relacionada con su parte hidrofóbica (ligando orgánico). Otra importante hipótesis indica que la acción antimicrobiana del MOF se relaciona con la liberación del ión metálico (Wyszogrodzka et al., 2016; Kumar et al., 2019). 


\section{Avances recientes e investigación futura en la liberación de fármacos empleando nanoMOFs}

Los MOFs han sido empleados como vehículos de fármacos desde 2006 debido a su alta porosidad, capacidad de almacenamiento, modulación de su estructura/propiedades y fácil funcionalización. Su baja toxicidad, fácil preparación de nanopartículas y relativa labilidad de los enlaces metal-ligando favorecen su biodegradabilidad. Además, son eficientes como agentes de contraste en imágenes de resonancia magnética, tomografía por computadora e imagenología óptica, como agentes sensores en terapia fototermal y fotodinámica, y como sistemas de liberación de fármacos (Cabrera-García et al., 2019).

En un trabajo reciente (Unamuno et al., 2018), se efectúo la encapsulación de gentamicina en dos MOFs biocompatibles de carboxilatos de hierro y zirconio mediante una simple impregnación. Consiguiendo una encapsulación reproducible de $600 \mu \mathrm{g}$ por mg de MOF, se obtienen nanopartículas mesoporosas de MOFs de $\mathrm{Fe}^{3+}$ y $\mathrm{Zr}^{4+}$.

Otra tendencia en el uso de MOF como sistema de liberación de fármacos para evitar su rápida degradación es su recubrimiento con biopolímeros, especialmente para el transporte de fármacos anticancerígenos. Javanbakht et al. (2019) reportan el encapsulamiento del fluoracil, un fármaco anticancerígeno, encapsulado en un MOF- 5 a base de $\mathrm{Zn}^{2+}$ mediante la impregnación del MOF en una solución del fármaco. Luego, el MOF-5 cargado con fluoracil fue recubierto de carboximetilcelulosa, cuya función fue formar una nanopartícula en estado hidrogel capaz de proteger al MOF del pH ácido en medios fisiológicos, efectuar la liberación controlada del fármaco, mejorar su estabilidad coloidal, permeabilidad y estabilidad mecánica (Wang et al., 2016; Nadar et al., 2019).

Por otro lado, la investigación a futuro en la liberación de fármacos empleando MOFs debe incorporar métodos de síntesis que no involucren el uso de solventes, que utilicen metales biocompatibles como $\mathrm{Ca}^{2+}, \mathrm{K}^{+}, \mathrm{Ti}^{4+}, \mathrm{Fe}^{3+}$, $\mathrm{Al}^{3+} \mathrm{y} \mathrm{Mo}^{6+}$. Además de utilizar ligandos orgánicos biológicamente aceptables como péptidos, carbohidratos, aminoácidos y derivados de la ciclodextrina para disminuir la citotoxicidad de sus productos de degradación (Rajkumar et al., 2019; Guo y Jiang, 2020).

El control de la cinética de liberación de un vehículo transportador de fármaco representa un reto mayor que solo el proceso de encapsulamiento. Para ello son necesarios más estudios de encapsulación/liberación de varios fármacos modelos para predecir el mejor MOF para un fármaco dado y el tiempo de liberación en condiciones determinadas para aplicaciones biomédicas.

El control de la degradación del MOF involucra su estabilidad y compatibilidad sanguínea, permitiendo su degradación hasta llegar a las células objetivo para la liberación controlada y sostenible del fármaco. Los sistemas en 
estado hidrogel basados en biopolímeros que incluyan MOFs nanoestructurados con terapéuticos adsorbidos representan una alternativa para modular estos requerimientos; los estudios in vitro de este tipo de materiales inteligentes podrían proporcionar evidencia de los mecanismos de liberación de los terapéuticos encapsulados y la evaluación de la respuesta biológica, mientras que estudios in vivo podrían asegurar la eficacia del terapéutico por el control de las propiedades de degradación, hinchamiento y mecánica que este sistema representaría. Por último, los MOFs representan un medio para hacer posible la liberación controlada de fármacos que aún no han sido comercializados debido a una baja solubilidad y estabilidad a condiciones fisiológicas (Horcajada et al., 2010; Wuttke et al., 2017), por lo cual estudiar estos sistemas de materiales representa un campo de investigación prometedor.

\section{Conclusiones}

Los MOFs nanoestructurados son materiales de naturaleza orgánica e inorgánica que combinan las propiedades de las nanopartículas inorgánicas como una alta capacidad de adsorción, estabilidad mecánica y las propiedades intrínsecas de las nanopartículas (eléctricas, ópticas y magnéticas); junto con las propiedades de las nanopartículas orgánicas como biodegradabilidad, baja citotoxicidad y actividad antimicrobiana regulada. Todas estas características convierten a los MOFs en un material ideal para la liberación controlada y dirigida de fármacos. A la fecha, es necesario diseñar sistemas terapéuticos basados en MOFs para asegurar la eficacia biomédica, lo cual puede lograrse controlando su estructura y propiedades. Específicamente, la regulación de la capacidad de liberación controlada de un fármaco adsorbido en un MOF debe incluir asegurar la internalización celular, lo cual está asociado con la estructura orgánica, y que los subproductos de degradación no presenten carácter citotóxico, algo que puede ser regulado usando cationes metálicos y ligandos biocompatibles. Por lo tanto, el desarrollo de este tipo de materiales representa una alternativa con potencial aplicación en estrategias de biomedicina.

\section{Referencias}

Abanades Lázaro, Isabel, Ross S. Forgan. (2019). Application of zirconium MOFs in drug delivery and biomedicine. Coordination Chemistry Reviews, 380: 230-259. http://doi.org/10.1016/j.ccr.2018.09.009

Amini-Fazl, Mohammad, Reza Mohammadi, Karim Kheiri. (2019). 5-Fluorouracil loaded chitosan/polyacrylic acid/ $\mathrm{Fe}_{3} \mathrm{O}_{4}$ magnetic nanocomposite hydrogel as a potential anticancer drug delivery system. International Journal of Biological Macromolecules, 132: 506-513. http://doi.org/10.1016/j.ijbiomac.2019.04.005

An, Hongde, Mingmin Li, Jia Gao, Zhenjie Zhang, Shengqian Ma, Yao Chen. (2019). Incorporation of biomolecules in metal-organic frameworks for advanced appli- 
cations. Coordination Chemistry Reviews, 384: 90-106. https://doi.org/10.1016/j. ccr.2019.01.001

Angelos, Sarah, Monty Liong, Eunshil Choi, Jeffrey I. Zink. (2008). Mesoporous silicate materials as substrates for molecular machines and drug delivery. Chemical Engineering Journal, 137(1): 4-13. https://doi.org/10.1016/j.cej.2007.07.074

Anirudhan, T. S., J. Christa. (2020). Temperature and pH sensitive multi-functional magnetic nanocomposite for the controlled delivery of 5-fluorouracil, an anticancer drug. Journal of Drug Delivery Science and Technology, 55: 101476. https://doi.org/10.1016/j.jddst.2019.101476

Bashir, Sajid, Sai Raghuveer Chava, Daqiang Yuan, Srinath Palakurthi, Jingbo Liu. (2020). Chapter 16 - Metal-organic frameworks and exemplified cytotoxicity evaluation. En Masoud Mozafari (ed.), Metal-organic frameworks for biomedical applications. Woodhead Publishing, 347-381. https://doi.org/10.1016/B9780-12-816984-1.00018-4

Banerjee, Sayan, Christina T. Lollar, Zhifeng Xiao, Yu Fang, Hong-Cai Zhou. (2020). Biomedical integration of metal-organic frameworks. Trends in Chemistry, 2(5): 467-479. https://doi.org/10.1016/j.trechm.2020.01.007

Barclay, Thomas G., Candace Minhthu Day, Nikolai Petrovsky, Sanjay Garg. (2019). Review of polysaccharide particle-based functional drug delivery. Carbohydrate Polymers, 221: 94-112. https://doi.org/10.1016/j.carbpol.2019.05.067

Beg, Sarwar, Mahfoozur Rahman, Atul Jain, Sumant Saini, Patrick Midoux, Chantal Pichon, Farhan Ahmad, Sohail Akhter, Sohail. (2017). Nanoporous metal organic frameworks as hybrid polymer-metal composites for drug delivery and biomedical applications. Drug Discovery Today, 22(4): 625-637. https://doi. org/10.1016/j.drudis.2016.10.001

Bigham, Ashkan, S. A. Hassanzadeh-Tabrizi, Mohammad Rafienia, Hossein Salehi. (2016). Ordered mesoporous magnesium silicate with uniform nanochannels as a drug delivery system: The effect of calcination temperature on drug delivery rate. Ceramics International, 42(15): 17185-17191. https://doi.org/10.1016/j. ceramint.2016.08.009

Bruch, Gisele E., Lorena F. Fernandes, Beatriz L. T. Bassi, Marco Túllio R. Alves, Isabelle O. Pereira, Frédéric Frézard, André R. Massensini. (2019). Liposomes for drug delivery in stroke. Brain Research Bulletin, 152: 246-256. https://doi. org/10.1016/j.brainresbull.2019.07.015

Bunker, Alex, Aniket Magarkar, Tapani Viitala. (2016). Rational design of liposomal drug delivery systems, a review: Combined experimental and computational studies of lipid membranes, liposomes and their PEGylation. Biochimica et Biophysica Acta (BBA) - Biomembranes, 1858(10): 2334-2352.

https://doi.org/10.1016/j.bbamem.2016.02.025

Cabrera-García, Alejandro, Elisa Checa-Chavarria, Eva Rivero-Buceta, Victoria Moreno, Eduardo Fernández, Pablo Botella. (2019). Amino modified metal-organic frameworks as $\mathrm{pH}$-responsive nanoplatforms for safe delivery of camptothecin. Journal of Colloid and Interface Science, 541: 163-174. https://doi.org/10.1016/j. jcis.2019.01.042 
Mundo Nano | ARTículos DE REVISIón | www.mundonano.unam.mx

14(26), 1e-29e, enero-junio 2021 | https:/ / doi.org/10.22201/ceiich.24485691e.2021.26.69634

Jesús A. Claudio-Rizo, Lucia F. Cano Salazar, Tirso E. Flores-Guia, Denis A. Cabrera-Munguia

Cai, Hong, Yong-Liang Huang, Dan Li. (2019). Biological metal-organic frameworks: Structures, host-guest chemistry and bio-applications. Coordination Chemistry Reviews, 378: 207-221. https://doi.org/10.1016/j.ccr.2017.12.003

Couvreur, Patrick. (2012). Nanoparticles in drug delivery: Past, present and future. Advanced Drug Delivery Reviews, 65(1): 3-21. http://doi.org/10.1016/j. addr.2012.04.010

Christodoulou, Ioanna, Christian Serre, Ruxandra Gref. (2020). Chapter 21 Metal-organic frameworks for drug delivery: Degradation mechanism and in vivo fate. En Masoud Mozafari (ed.), Metal-organic frameworks for biomedical applications. Woodhead Publishing, 467-489. https://doi.org/10.1016/B978-0-12-816984-1.00023-8

Dong, Peng, K. P. Rakesh, H. M. Manukumar, Yasser Hussein Eissa Mohammed, C. S. Karthik, S. Sumathi, P. Mallu, Hua-Li Qin. (2019). Innovative nano-carriers in anticancer drug delivery-a comprehensive review. Bioorganic Chemistry, 85: 325-336. https://doi.org/10.1016/j.bioorg.2019.01.019

Duan, Qianqian, Lan Ma, Boye Zhang, Yixia Zhang, Xiaoning Li, Tao Wang, Wendong Zhang, Yi Li, Shengbo Sang. (2019). Construction and application of targeted drug delivery system based on hyaluronic acid and heparin functionalised carbon dots. Colloids and Surfaces B: Biointerfaces, 188: 110768. https://doi. org/10.1016/j.colsurfb.2019.110768

Evans, Jack D., Bikash Garai, Helge Reinsch, Weijin Li, Stefano Dissegna, Volodymyr Bon, Irena Senkovska, Roland A. Fischer, Stefan Kaskel, Christoph Janiak, Norbert Stock, Dirk Volkmer, (2019). Metal-organic frameworks in Germany: From synthesis to function. Coordination Chemistry Reviews, 380: 378-418. https://doi.org/10.1016/j.ccr.2018.10.002

Forouzandehdel, Shayan, Sherwin Forouzandehdel, Mina Rezghi Rami. (2020). Synthesis of a novel magnetic starch-alginic acid-based biomaterial for drug delivery. Carbohydrate Research, 487: 107889. https://doi.org/10.1016/j.carres.2019.107889

Gaudin, C., D. Cunha, E. Ivanoff, P. Horcajada, G. Chevé, A. Yasri, O. Loget, C. Serre, G. Maurin. (2012). A quantitative structure activity relationship approach to probe the influence of the functionalization on the drug encapsulation of porous metal-organic frameworks. Microporous and Mesoporous Materials, 157: 124-130. https://doi.org/10.1016/j.micromeso.2011.06.011

Giménez-Marqués, M., T. Hidalgo, C. Serre, P. Horcajada, (2016). Nanostructured metal-organic frameworks and their bio-related applications. Coordination Chemistry Reviews, 307(2): 342-360. https://doi.org/10.1016/j.ccr.2015.08.008

Gulcay, Ezgi, Ilknur, Erucar. (2020). 6. Metal-organic frameworks for biomedical applications. En Raju Khan, Shaswat Barua (eds.), Two-dimensional nanostructures for biomedical technology, 173-210. https://doi.org/10.1016/B978-0-12-8176504.00006-1

Guo, Qin, Chen Jiang (2020). Delivery strategies for macromolecular drugs in cancer therapy. Acta Pharmaceutica Sinica B. https://doi.org/10.1016/j.apsb.2020.01.009

He, Haisheng, Yi Lu, Jianping Qi, Quangang Zhu, Zhongjian Chen, Wei Wu. (2019). Adapting liposomes for oral drug delivery. Acta Pharmaceutica Sinica B, 9(1): 36-48. https://doi.org/10.1016/j.apsb.2018.06.005 
Horcajada, Patricia, Tamim Chalati, Christian Serre, Brigitte Gillet, Catherine Sébrié, Tarek Baati, Jarrod Eubank, Daniela Heurtaux, Pascal Clayette, Christine Kreuz, Jong-San Chang, Young Kyu Hwang, Veronique Marsaud, Phuong-Nhi Bories, Luc Cynober, Sophie Gil, Gerard Férey, Patrick Couvreur, Ruxandra Gref. (2010). Porous metal-organic-framework nanoscale carriers as a potential platform for drug delivery and imaging. Nature materials, 9: 8-172. https:// doi.org/10.1038/nmat2608

Huang, Da, Decheng Wu. (2018). Biodegradable dendrimers for drug delivery. Materials Science and Engineering: C, 90:713-727. https://doi.org/10.1016/j. msec.2018.03.002

Huxford, Rachel C., Joseph Della Rocca, Wenbin Lin. (2010). Metal-organic frameworks as potential drug carriers. Current Opinion in Chemical Biology, 14(2): 262-268. https://doi.org/10.1016/j.cbpa.2009.12.012

Javanbakht, Siamak, Afsaneh Hemmati, Hassan Namazi, Abolfazl Heydari. (2019). Carboxymethyl cellulose-coated 5-fluorouracil@MOF-5 nano-hybrid as a bionanocomposite carrier for the anticancer oral delivery. International Journal of Biological Macromolecules. https://doi.org/10.1016/j.ijbiomac.2019.12.007

Kaur, Jashandeep, Gurlal Singh Gill, Kiran Jeet. (2019). Chapter 5 - Applications of carbon nanotubes in drug delivery: A comprehensive review. En Shyam S. Mohapatra, Shivendu Ranjan, Nandita Dasgupta, Raghvendra Kumar Mishra, Sabu Thomas (eds.), Micro and nano technologies, characterization and biology of nanomaterials for drug delivery, 113-135. https://doi.org/10.1016/B978-0-12814031-4.00005-2

Khan, Nazmul Abedin, Zubair Hasan, Sung Hwa Jhung. (2018). Beyond pristine metal-organic frameworks: Preparation and application of nanostructured, nanosized and analogous MOFs. Coordination Chemistry Reviews, 376: 20-45. https://doi.org/10.1016/j.ccr.2018.07.016

Kumar, Pawan, Bhaskar Anand, Yiu Fai Tsang, Ki-Hyun Kim, Sadhika Khullar, Bo Wang. (2019). Regeneration, degradation, and toxicity effect of MOFs: Opportunities and challenges. Environmental Research, 176: 108488. https://doi. org/10.1016/j.envres.2019.05.019

Lakshmi, Buddolla Anantha, Sanghyo Kim. (2019). Current and emerging applications of nanostructured metal-organic frameworks in cancer-targeted theranostics. Materials Science and Engineering: C, 105: 110091. https://doi.org/10.1016/j. msec.2019.110091

Li, Chong, Jiancheng Wang, Yiguang Wang, Huile Gao, Gang Wei, Yongzhuo Huang, Haijun Yu, Yong Gan, Yongjun Wang, Lin Mei, Huabing Chen, Haiyan Hu, Zhiping Zhang, Yiguang Jin. (2019). Recent progress in drug delivery. Acta Pharmaceutica Sinica B, 9(6): 1145-1162. https://doi.org/10.1016/j.apsb.2019.08.003

Li, Sheng, Jun Zhang, Dan-Dan Ju, Xin Li, Jun-Cheng Zhang, Xu Yan, Hong-Di Zhang, Feng Song, Yun-Ze Long. (2018). Flexible inorganic composite nanofibers with carboxyl modification for controllable drug delivery and enhanced optical monitoring functionality. Chemical Engineering Journal, 350: 645-652, https://doi.org/10.1016/j.cej.2018.05.166 
Li, Zixian, Andre Luis Branco de Barros, Daniel Cristian Ferreira Soares, Sara Nicole Moss, Laleh Alisaraie. (2017). Functionalized single-walled carbon nanotubes: cellular uptake, biodistribution and applications in drug delivery. International Journal of Pharmaceutics, 524(1-2): 41-54. https://doi.org/10.1016/j.ijpharm.2017.03.017

Liu, Weicong., Yuyu Zhong, Xiaoxiong Wang, Canfeng Zhuang, Junhao Chen, Dong Liu, Weiwei Xiao, Ying Pan, Jianjing Huang, Jianqiang Liu. (2019). A porous $\mathrm{Cu}$ (II)-based metal-organic framework carrier for $\mathrm{pH}$-controlled anticancer drug delivery. Inorganic Chemistry Communications, 111: 107675. https://doi. org/10.1016/j.inoche.2019.107675

Lu, Yu-Jen, Er-Yuan Chuang, Yu-Hsin Cheng, T.S. Anilkumar, Huai-An Chen, Jyh-Ping Chen. (2019). Thermosensitive magnetic liposomes for alternating magnetic field-inducible drug delivery in dual targeted brain tumor chemotherapy. Chemical Engineering Journal, 373:720-733. https://doi.org/10.1016/j.cej.2019.05.055

Maleki, Reza, Hamid Hassanzadeh Afrouzi, Mirollah Hosseini, Davood Toghraie, Sara Rostami. (2020). Molecular dynamics simulation of Doxorubicin loading with $\mathrm{N}$-isopropyl acrylamide carbon nanotube in a drug delivery system. Computer Methods and Programs in Biomedicine, 184: 105303. https://doi.org/10.1016/j. cmpb.2019.105303

Mohammed, Razeeth Shait Mohammed, Varish Ahmad, Abrar Ahmad, Shams Tabrez, Hani Choudhry, Mazin A. Zamzami, Muhammed A. Bakhrebah, Aftab Ahmad, Samina Wasi, Hasan Mukhtar, Mohammad Imran Khan. (2019). Prospective of nanoscale metal organic frameworks [NMOFs] for cancer therapy. Seminars in Cancer Biology. https://doi.org/10.1016/j.semcancer.2019.12.015

Nadar Shamraja S., Leena Vaidya, Shefali Maurya, Virendra K. Rathod. (2019). Polysaccharide based metal organic frameworks (polysaccharide-MOF): A review. Coordination Chemistry Reviews, 396: 1-21. https://doi.org/10.1016/j.ccr.2019.05.011

Nadizadeh, Zahra, M. Reza Naimi-Jamal, Leila Panahi. (2018). Mechanochemical solvent-free in situ synthesis of drug-loaded $\{\mathrm{Cu} 2(1,4-\mathrm{bdc}) 2($ dabco) $\} \mathrm{n}$ MOFs for controlled drug delivery. Journal of Solid State Chemistry, 259: 35-42. https://doi.org/10.1016/j.jssc.2017.12.027

Nguyen Thi Thanh Uyen, Zuratul Ain Abdul Hamid, Nguyen Xuan Thanh Tram, Nurazreena Ahmad. (2020). Fabrication of alginate microspheres for drug delivery: A review. Int. J. Biol. Macromol., 153: 1035. https://doi.org/10.1016/j. ijbiomac.2019.10.233

Pander, Marzena, Anna Żelichowska, Wojciech Bury. (2018). Probing mesoporous Zr-MOF as drug delivery system for carboxylate functionalized molecules. Polyhedron, 156: 131-137. https://doi.org/10.1016/j.poly.2018.09.006

Pandey, Abhijeet, Namdev Dhas, Prashant Deshmukh, Carlos Caro, Pravin Patil, Maria Luisa García-Martín, Bharath Padya, Ajinkya Nikam, Tejal Mehta, Srinivas Mutalik. (2020). Heterogeneous surface architectured metal-organic frameworks for cancer therapy, imaging, and biosensing: A state-of-the-art review. Coordination ChemistryReviews, 409: 213212.https://doi.org/10.1016/j.ccr.2020.213212

Parker, Christina L., McSweeney Morgan D., Lucas Andrew T., Jacobs Timothy M., Wadswort Daniel, Zamboni William C., Lai Samuel K. (2019). Pretargeted deliv- 
ery of PEG-coated drug carriers to breast tumors using multivalent, bispecific antibody against polyethylene glycol and HER2. Nanomed-Nanotechnol, 21: 102076. https://doi.org/10.1016/j.nano.2019.102076

Paul, Willi, Chandra Sharma. (2010). Chapter 13 Inorganic nanoparticles for targeted drug delivery. En Chandra P. Sharma (ed.), Biointegration of medical implant materials: Science and design. Woodhead Publishing Series in Biomaterials, 204-235. http://doi.org/10.1533/9781845699802.2.204

Prajapati, Shiv Kumar, Ankit Jain, Aakanchha Jain, Sourabh Jain. (2019). Biodegradable polymers and constructs: A novel approach in drug delivery. European Polymer Journal, 120: 109191. https://doi.org/10.1016/j.eurpolymj.2019.08.018

Qian, Ken K., Robin H. Bogner. (2012). Application of mesoporous silicon dioxide and silicate in oral amorphous drug delivery systems. Journal of Pharmaceutical Sciences, 101(2): 444-463. https://doi.org/10.1002/jps.22779

Rajkumar, T., Deepak Kukkar, Ki-Hyun Kim, Jong Ryeul Sohn, Akash Deep. (2019). Cyclodextrin-metal-organic framework (CD-MOF): From synthesis to applications. Journal of Industrial and Engineering Chemistry, 72: 50-66. https://doi. org/10.1016/j.jiec.2018.12.048

Raphey, V. R., T. K. Henna, K. P. Nivitha, P. Mufeedha, Chinnu Sabu, K. Pramod. (2019). Advanced biomedical applications of carbon nanotube. Materials Science and Engineering: C, 100: 616-630. https://doi.org/10.1016/j.msec.2019.03.043

Rasheed, T., F. Nabeel, A. Raza, M. Bilal, H. M. N. Iqbal. (2019). Biomimetic nanostructures/cues as drug delivery systems: A review. Materials Today Chemistry, 13: 147-157. https://doi.org/10.1016/j.mtchem.2019.06.001

Ren, Jianwei, Xoliswa Dyosiba, Nicholas Musyoka, Henrietta W. Langmi, Mkhulu Mathe, Shijun Liao. (2017). Review on the current practices and efforts towards pilot-scale production of metal-organic frameworks (MOFs). Coordination Chemistry Reviews, 352: 187-219. http://doi.org/10.1016/j.ccr.2017.09.005

Rojas, Sara, Ana Arenas-Vivo, Patricia Horcajada. (2019). Metal-organic frameworks: A novel platform for combined advanced therapies. Coordination Chemistry Reviews, 388: 202-226. https://doi.org/10.1016/j.ccr.2019.02.032

Safaei, Mohadeseh, Mohammad Mehdi Foroughi, Nasser Ebrahimpoor, Shohreh Jahani, Ali Omidi, Mehrdad Khatami. (2019). A review on metal-organic frameworks: Synthesis and applications. Trends in Analytical Chemistry, 118: 401-425. https://doi.org/10.1016/j.trac.2019.06.007

Sharma, Suraj, Sweet Naskar, Ketousetuo Kuotsu (2019). A review on carbon nanotubes: Influencing toxicity and emerging carrier for platinum based cytotoxic drug application. Journal of Drug Delivery Science and Technology, 51: 708-720. https://doi.org/10.1016/j.jddst.2019.02.028

Sherje, Atul P., Mrunal Jadhav, Bhushan R. Dravyakar, Darshana Kadam. (2018). Dendrimers: A versatile nanocarrier for drug delivery and targeting. International Journal of Pharmaceutics, 548(1): 707-720. https://doi.org/10.1016/j. ijpharm.2018.07.030

Shijun, Tai, Weiquan Zhang, Jinsheng Zhang, Genxiang Luo, Yu Jia, Mingli Deng, Yun Ling. (2016). Facile preparation of UiO-66 nanoparticles with tunable sizes in a 
continuous flow microreactor and its application in drug delivery. Microporous and Mesoporous Materials, 220: 148-154. https://doi.org/10.1016/j.micromeso.2015.08.037

Unamuno, X, E. Imbuluzqueta, F. Salles, P. Horcajada, M. J. Blanco-Prieto. (2018). Biocompatible porous metal-organic framework nanoparticles based on Fe or Zr for gentamicin vectorization. European Journal of Pharmaceutics and Biopharmaceutics, 132: 11-18. https://doi.org/10.1016/j.ejpb.2018.08.013

Vahed, Tahereh Azizi, M. Reza Naimi-Jamal, Leila Panahi. (2019). Alginate-coated ZIF-8 metal-organic framework as a green and bioactive platform for controlled drug release. Journal of Drug Delivery Science and Technology, 49: 570576. https://doi.org/10.1016/j.jddst.2018.12.022

Wang, Julia, Jonah A. Kaplan, Yolonda Colson, Mark Grinstaff. (2016). Mechanoresponsive materials for drug delivery: Harnessing forces for controlled release. Advanced Drug Delivery Reviews, 108: 68-82. http://www.org/10.1016/j.addr.2016.11.001

Wang, Lei, Min Zheng, Zhigang Xie. (2018). Nanoscale metal-organic frameworks for drug delivery: A conventional platform with new promise. Journal of Materials Chemistry B, 6: 707-717. https://doi.org/10.1039/C7TB02970E

Wang, Ying, Yan Jianhua, Wen Nachuan, Xiong Hongjie, Shundong Cai, He Qunye, Hu Yaqin, Peng Dongming, Liu Zhenbao, Liu Yanfei. (2019). Metal-organic frameworks for stimuli-responsive drug delivery. Biomaterials, 230: 119619. https://doi.org/10.1016/j.biomaterials.2019.119619

Wang, Zhe, Xiangping Deng, Jinsong Ding, Wenhu Zhou, Xing Zheng, Guotao Tang. (2017). Mechanisms of drug release in pH-sensitive micelles for tumor targeted drug delivery system: A review. International Journal of Pharmaceutics, 535(1-2): 253-260. https://doi.org/10.1016/j.ijpharm.2017.11.003

Wei, Shi, Yern Chee Ching, Cheng Hock Chuah. (2020). Synthesis of chitosan aerogels as promising carriers for drug delivery: A review. Carbohydrate Polymers, 231: 115744. https://doi.org/10.1016/j.carbpol.2019.115744

Wuttke, Stefan, Marjorie Lismont, Alberto Escudero, Bunyarat Rungtaweevoranit, Wolfgang J. Parak. (2017). Positioning metal-organic framework nanoparticles within the context of drug delivery - A comparison with mesoporous silica nanoparticles and dendrimers. Biomaterials, 123: 172-183.

https://doi.org/10.1016/j.biomaterials.2017.01.025

Wyszogrodzka, Gabriela, Bartosz Marszałek, Barbara Gil, Przemysław Dorożyński, (2016). Metal-organic frameworks: mechanisms of antibacterial action and potential applications. Drug Discovery Today, 21(6): 1009-1018. https://doi. org/10.1016/j.drudis.2016.04.009

Xu, Min, Nan Li. (2020). Chapter 12 - Metal-based nanocontainers for drug delivery in tumor therapy. En Phuong Nguyen-Tri, Trong-On Do, Tuan Anh Nguyen (eds.), Smart nanocontainers: In micro and nano technologies, 195-215. https:// doi.org/10.1016/B978-0-12-816770-0.00012-5

Yao, Qingqing, Liu Yangxi, Selvaratnam Balaranjan, Koodali Ranjit, Sun Hongli. (2018). Mesoporous silicate nanoparticles/3D nanofibrous scaffold-mediated dual-drug delivery for bone tissue engineering. Journal of Controlled Release, 
279: 69-78. http://www.doi.org/10.1016/j.jconrel.2018.04.011

Zhang, Lei, Yan Chen, Rui Shi, Tingguo Kang, Guangsheng Pang, Boran Wang, Yue Zhao, Xu Zeng, Changxin Zou, Peng Wu, Jiayang Li. (2018). Synthesis of hollow nanocages MOF-5 as drug delivery vehicle to solve the load-bearing problem of insoluble antitumor drug oleanolic acid (OA). Inorganic Chemistry Communications, 96: 20-23. https://doi.org/10.1016/j.inoche.2018.07.029

Zhang, Shu, Xibo Pei, Huile Gao, Song Chen, Jian Wang. (2020). Metal-organic framework-based nanomaterials for biomedical applications. Chinese Chemical Letters, 31(5): 1060-1070. https://doi.org/10.1016/j.cclet.2019.11.036

Zhang, Ying, Hon Fai Chan, Kam W. Leong. (2013). Advanced materials and processing for drug delivery: The past and the future. Advanced Drug Delivery Reviews, 65(1): 104-120. https://doi.org/10.1016/j.addr.2012.10.003

Zhang, Zhan, Wei Sang, Lisi Xie, Yunlu Dai. (2019). Metal-organic frameworks for multimodal bioimaging and synergistic cancer chemotherapy. Coordination Chemistry Reviews, 399: 213022. https://doi.org/10.1016/j.ccr.2019.213022

Zheng, Yilin, Li Ziying, Chen Haijun, Gao Yu. (2020). Nanoparticle-based drug delivery systems for controllable photodynamic cancer therapy. European Journal of Pharmaceutical Sciences, 144: 105213. http://doi.org/10.1016/j.ejps.2020.105213

Zhong, Xiaofang, Yunting Zhang, Lu Tan, Tao Zheng, Yingying Hou, Xiaoyu Hong, Guangsheng Du, Xiaoyan Chen, Yuandong Zhang, Xun Sun. (2019). An aluminum adjuvant-integrated nano-MOF as antigen delivery system to induce strong humoral and cellular immune responses. Journal of Controlled Release, 300: 81-92. https://doi.org/10.1016/j.jconrel.2019.02.035

Zhu, Wenjun, Jiayue Zhao, Qian Chen, Zhuang Liu. (2019). Nanoscale metal-organic frameworks and coordination polymers as theranostic platforms for cancer treatment. Coordination Chemistry Reviews, 398: 113009. https://doi.org/10.1016/j. ccr.2019.07.006 